\title{
The Added Benefit of Combining Laser Doppler Imaging With Clinical Evaluation in Determining the Need for Excision of Indeterminate-Depth Burn Wounds
}

Mohammed Asif ${ }^{1}$, An Guo Michael Chin ${ }^{2}$, Tomer Lagziel ${ }^{1,3}$, Kevin M. Klifto ${ }^{1}$, Ashley D. Modica ${ }^{4}$, Eliana Duraes $^{1}$, Julie Caffrey ${ }^{1}$, Charles S. Hultman ${ }^{1}$

1. Plastic Surgery, Johns Hopkins University School of Medicine, Baltimore, USA 2. General Surgery, St. John's Episcopal Hospital, Far Rockaway, USA 3. Medicine, Tel-Aviv University, Sackler School of Medicine, Tel-Aviv, ISR 4. Plastic Surgery, University of South Florida (USF) Health, Tampa, USA

Corresponding author: Mohammed Asif, masif2@jhmi.edu

\section{Abstract \\ Background}

Managing indeterminate-depth burn wounds remains challenging. Laser Doppler Imaging (LDI) has been validated for burn wound depth and can influence the clinical assessment. Our study investigated the value of LDI as an adjunct in determining the need for excision.

\section{Methods}

Seventy American Burn Association (ABA)-verified burn centers were surveyed. A controlled pre-test assessment without LDI and post-test assessment with LDI of 100 indeterminate-depth burn wounds was conducted to evaluate the influence on the clinical judgment among different health professionals. Relative risk, analysis of variance (ANOVA), paired t-test, and intention-to-treat were used for analysis. A p-value $\leq$ 0.05 was considered significant.

\section{Results}

Among 32 burn centers, three confirmed using LDI. Six thousand grader-image interactions were analyzed. There was a significant difference in the predictive accuracy for pre-LDI and post-LDI assessments when all graders were considered $(51.9 \% \pm 7.0$ vs. $72.9 \% \pm 7.9 ; \mathrm{p}<0.0001)$. Post-LDI assessment added $20.9 \%$ more accuracy than the pre-LDI assessment. The post-LDI assessment was 1.4 times more likely to correctly predict the need for excision and skin-grafting than the pre-LDI assessment. All groups had an improved performance post-LDI: Group 1 (physicians), $51.9 \pm 7.5$ versus $76.4 \pm 5$; Group 2 (nurses), $52.1 \pm 6.1$ versus $72.7 \pm 7.7$; and Group 3 (others), $51.7 \pm 9.2$ versus $68.6 \pm 10.1$. No statistical difference was observed between groups $(\mathrm{p}=0.92)$.

Received 06/03/2020

Review began 06/11/2020 Review ended 06/17/2020 Published 06/22/2020

๑) Copyright 2020 Asif et al. This is an open access article distributed under the terms of the Creative Commons Attribution License CC-BY 4.0., which permits unrestricted use, distribution, and reproduction in any medium, provided the original author and source are credited.

\section{Conclusion}

LDI makes the clinical examination of indeterminate-depth burn wounds more accurate. For every five LDI evaluations performed, one assessor changed their treatment plan as a result of this imaging technique. LDI is cost-effective and increases the accuracy of determining the severity of indeterminate-depth burn wounds.

Categories: Plastic Surgery, General Surgery, Trauma

Keywords: laser doppler imaging (ldi), indeterminate depth burn wounds, digital health communications, digital images, burn surgery, second degree burn, burn, wound care

\section{Introduction}

Accurate visual assessment of burns is an integral step in the treatment plan process for indeterminatedepth burn injuries. Yet, visual assessment of burns has remained relatively unchanged since the 16th century [1]. With the recent upsurge of telemedicine around the world, many healthcare providers are accepting the use of digital health communications in their medical practices [2]. Digital health communications are becoming especially popular in burn centers because of their improved convenience, accuracy, efficiency, productivity, and clinical decision-making [3]. Telemedicine is constantly explored for placements and improvements in healthcare settings, but its integration into practice can often be difficult to implement [4].

Traditionally, verbal or written burn wound descriptions were the sole source of communication between healthcare professionals with limitations of subjectivity, which hindered accuracy. Yet, when digital images were introduced, the accuracy of burn assessments improved dramatically [5]. It is more efficient to make an assessment from a digital image than from a provided wound description from other healthcare 


\section{Cureus}

professionals, especially non-burn specialists [2].

However, digital images are not perfect in assessing burn wounds since they lack important information such as burn depth. Like a naked eye, a digital image has its limitation of a two-dimensional view of a burn wound surface and cannot provide a visual of the deeper layers of the skin [6]. Two-dimensional (2D) digital photographs are limited because they have been shown to increase validity in size assessment, but not depth assessment [7]. Even though a 2D view of a burn wound can provide good information, having tools that provide additional dimensions of assessment would further improve clinical burn wound outcomes.

There have been numerous technological developments to assist in managing burn wounds, including Laser Doppler Imaging (LDI), which is a helium-neon laser that objectively measures the blood flow and/or healing potential for indeterminate-depth burn wounds. LDI is a non-invasive mechanical extension of the clinical physical exam for burn wounds because it objectively identifies burns that have good or poor wound healing potential to help decide whether or not to proceed with surgical excision and grafting.

Even though studies indicate that visual evaluation of digital images is accurate enough for clinical decisionmaking, there are not sufficient studies looking into the accuracy of assessing digital images of indeterminate-depth burn wounds [8]. The reliability of making a preliminary indeterminate-depth burn wound assessment based on digital images should be further explored due to its two-dimensional limitations.

We investigated how additional information from LDI can influence the clinical assessment of indeterminate-depth burn wound digital images. The aim of this study was to investigate the accuracy of burn wound photographs and the value of LDI as an adjunct to clinical evaluation in determining the need for excision of 100 indeterminate-depth burn wounds among different healthcare providers.

\section{Materials And Methods}

We conducted a controlled pre-test/post-test assessment at a burn center to evaluate the influence of LDI on the clinical judgment of physicians (Group 1), nurses and physician assistants (Group 2), and other health professionals (Group 3).

The study involved a retrospective review of 100 patients over three years and inclusion criteria included patients aged 18 years or older with indeterminate-depth burns who received an LDI scan. The controlled assessment consisted of regular digital images of 100 burn wounds for the pre-test, and the same images side-by-side with 100 LDI color-coded images for the post-test. LDI was performed, on average, 2.81 days after the injury was sustained. For burn wound management, the historical decision of two senior burn surgeons was considered standard of care. The determination of surgical versus non-surgical intervention was based on these surgeons' expert opinion after clinical and technological evaluation. A burn surgeon's expert opinion is a culmination of academic knowledge and professional, clinical experience. Relative risk, analysis of variance (ANOVA), paired t-test, and intention-to-treat were used for analysis. A p-value $\leq 0.05$ was considered significant (Figure 1).

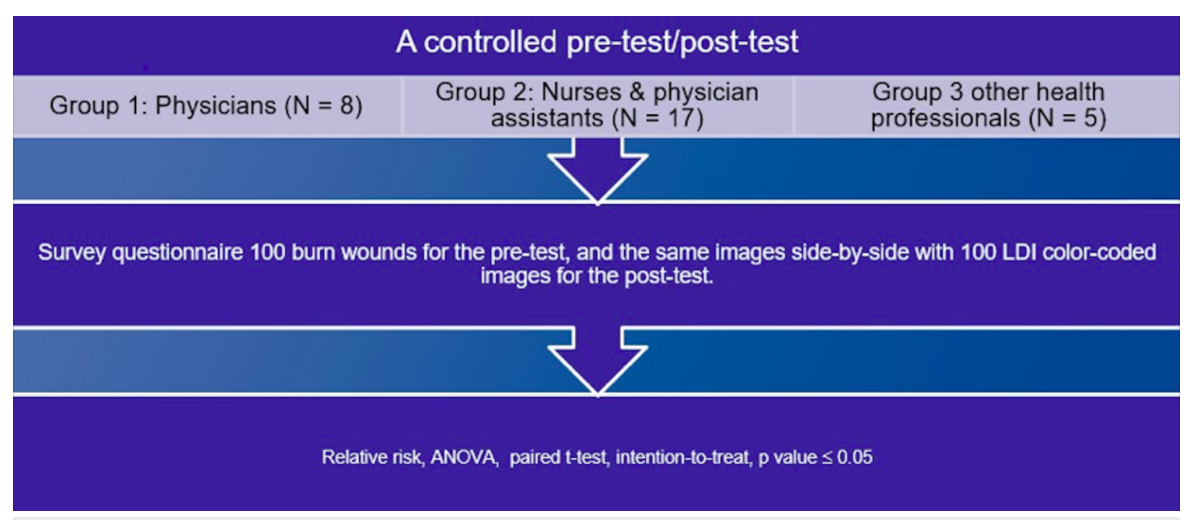

FIGURE 1: A controlled pre-test/post-test assessment algorithm ANOVA, analysis of variance; LDI, Laser Doppler Imaging

We also carried out a survey of 70 American Burn Association (ABA)-verified burn centers on the utilization of LDI methods (Figure 2). The survey was a basic questionnaire of ABA burn centers to understand if they implement LDI technology in their analysis and treatment plan of indeterminate-depth burn wounds. 


\section{Cureus}

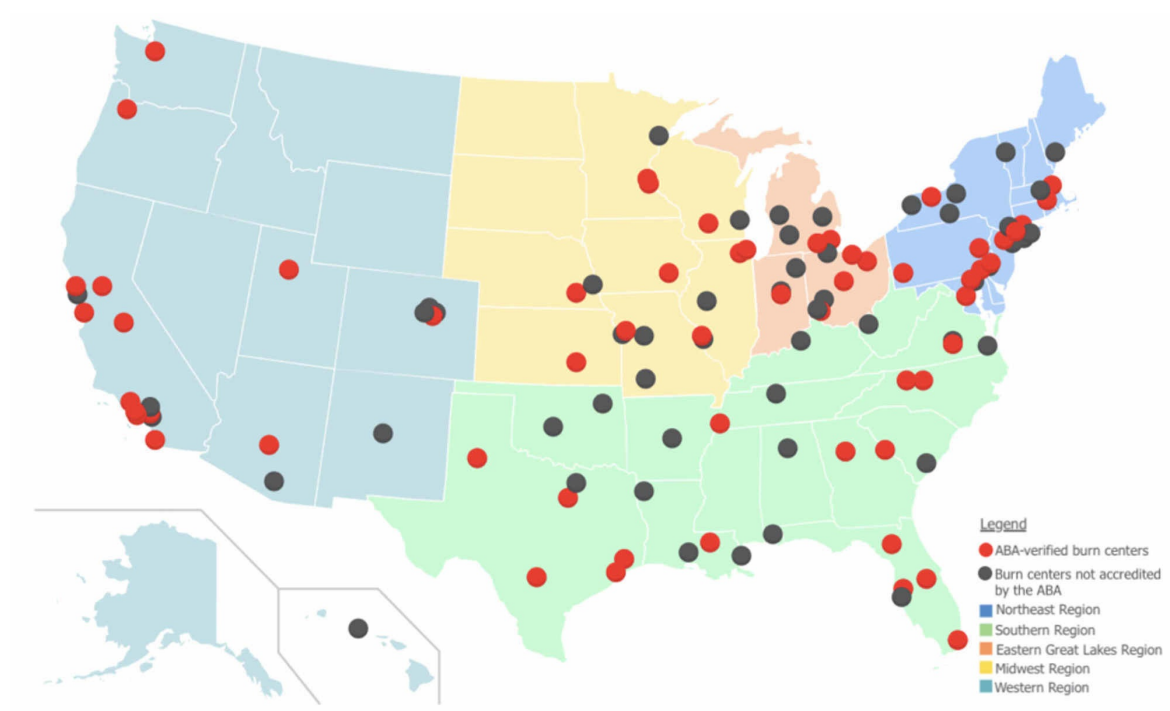

FIGURE 2: Map of ABA-verified burn centers across the United States

Adapted from the American Burn Association [9]

\section{Results}

A group of 30 professionals analyzed 100 digital images with and without LDI resulting in a total of 6,000 grader-image interactions were analyzed. There was a significant difference in the predictive accuracy for pre-LDI and post-LDI assessments when all graders were considered ( $51.9 \% \pm 7.0$ vs. $72.9 \% \pm 7.9 ; \mathrm{p}<0.0001)$ (Figure 3). On average, the post-LDI assessment was $20.9 \%$ more accurate than the pre-LDI assessment (95\% CI [17.388, 24.478]). The post-LDI assessment was 1.4 times ( $\mathrm{RR}=1.4$, CI [1.12-1.75]) more likely to correctly predict the need for excision and skin grafting than the pre-LDI assessment alone. All professional groups had an improved performance post-LDI: Group 1, $51.9 \pm 7.5$ versus $76.4 \pm 5$; Group 2, $52.1 \pm 6.1$ versus $72.7 \pm$ 7.7 ; and Group 3, $51.7 \pm 9.2$ versus $68.6 \pm 10.1$; no statistical difference was observed between groups $(p=$ 0.92) (Figures 4, 5).

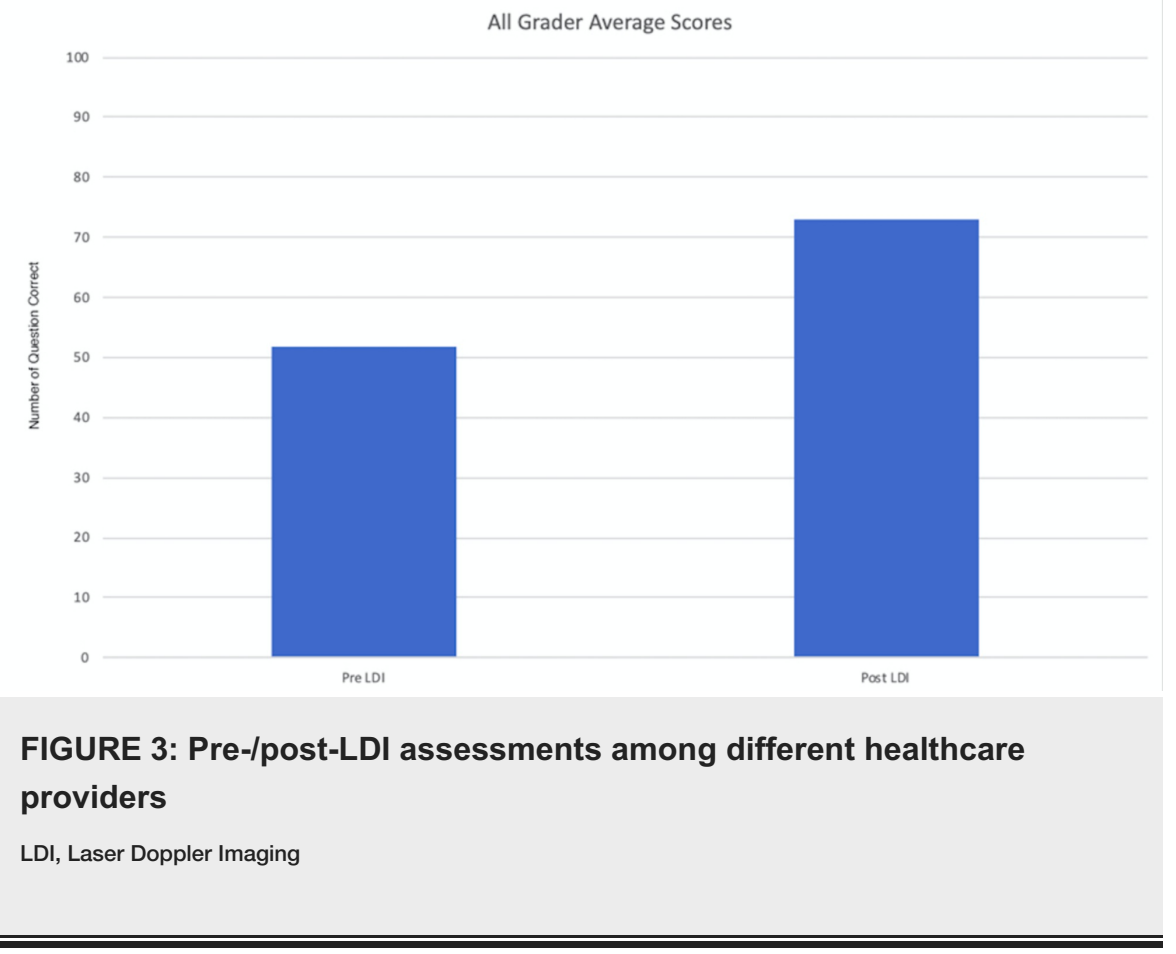




\section{Cureus}

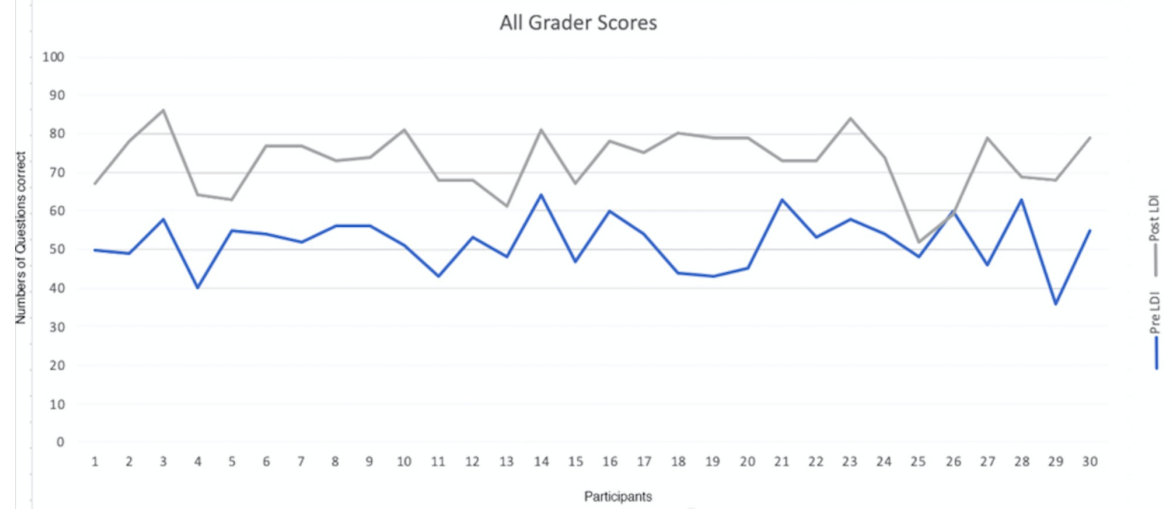

FIGURE 4: Post-LDI assessment improved among different healthcare providers

LDI, Laser Doppler Imaging

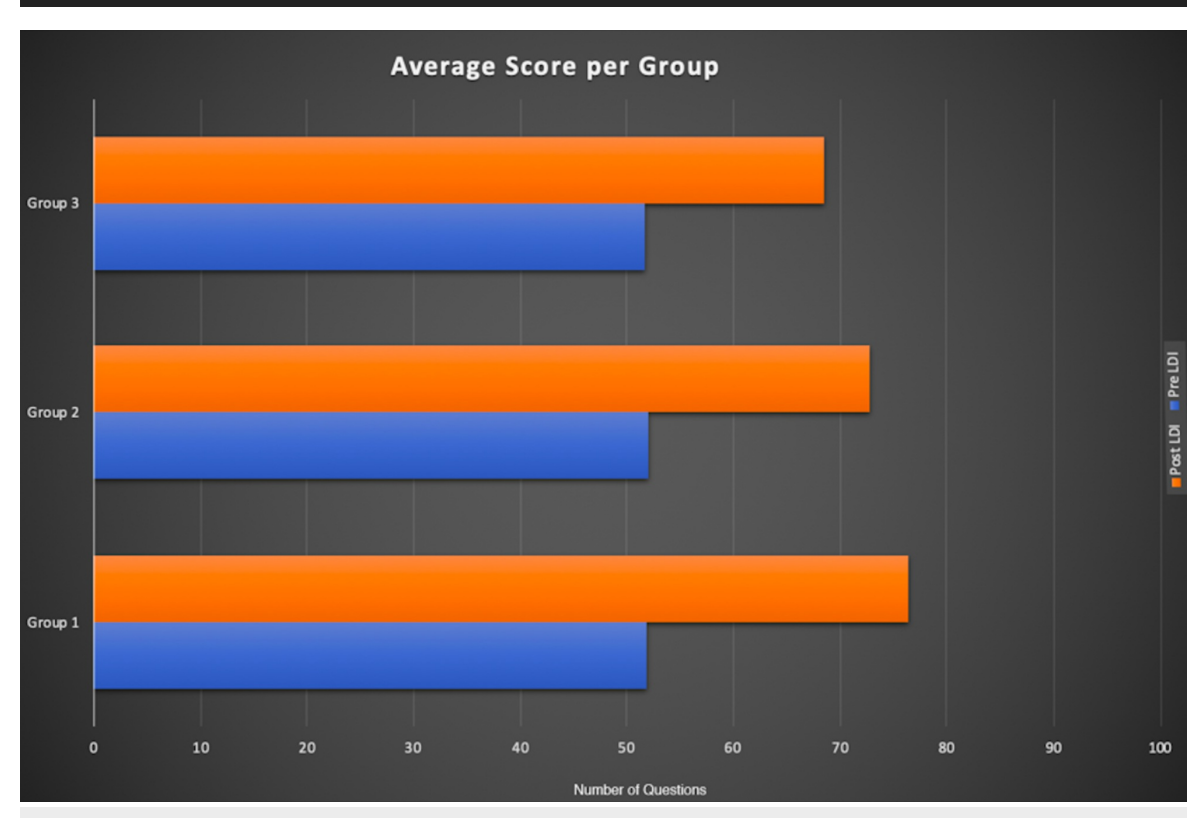

FIGURE 5: Breakdown of pre-/post-LDI assessments among different healthcare providers

LDI, Laser Doppler Imaging

Survey results demonstrated that of the $32 \mathrm{ABA}$-verified burn centers who responded, only five confirmed using supplemental digital technology, three of which were LDI. The other modalities were infrared spectroscopy and thermography.

\section{Discussion}

The field of burn surgery has evolved slowly in the past century when comparing to other surgical specialties as evident by our telephonic survey. There were only five ABA-verified burn centers that used supplemental technology to adjunct their clinical assessment of the indeterminate-depth burn wounds. Our study demonstrated that the use of high-resolution JPEG images yielded accurate results in only $51.9 \%$ of the cases and after evaluating the same images with LDI assessment, the predictive accuracy improved $20.9 \%$ among different health providers. Notably, for every five LDI evaluations, one assessor changed their treatment plan in response to LDI, resulting in less surgical interventions, decreased length of stay, less opioid prescriptions, and lower overall morbidity. In 2018, a study published by King Edward Medical University and Mayo Hospital in Pakistan comparing the accuracy of LDI and clinical assessment in determining wound depth showed $90.21 \%$ diagnostic accuracy, $92.75 \%$ sensitivity, $82 \%$ specificity, $94 \%$ positive predictive value, and $79 \%$ negative predictive value. The results for clinical assessment were $81.52 \%, 81 \%$, $82 \%, 93 \%$, and $59 \%$, respectively [8]. Burn surgery and wound management involve a multidisciplinary 
treatment team. This highlights the importance of our methodology involving review by non-physicians and physicians. The standard non-LDI approach involves a more aggressive and less precise surgery. The change resulted from LDI intervention involved either a non-invasive treatment approach or a more targeted surgery. The more effective healing allowed for a shorter hospital stay.

The majority of developments in the field have occurred relatively recently, due to an increase in burn research, better understanding of wound physiology, and new surgical and critical care techniques [10]. Many technologies are emerging for burn care with LDI at the forefront of clinical burn depth assessment, having the ability to evaluate microvascular dermal perfusion [11]. To highlight its effectiveness, LDI has even been validated in a multi-center study, examining over 400 wounds and yielding $96 \%$ technical accuracy in predicting healing time [12]. Other studies that have compared LDI and other imaging technologies have demonstrated significantly more accurate results with LDI [13].

The primary competitor to LDI in the field of digital imaging assessment is thermal imaging technology. This not-so-modern technology dates back to the 1960s and maps skin temperatures using infrared scanning techniques [14]. Today, LDI and thermal imaging are the two primary adjuncts to assist in clinical assessments of indeterminate burns due to their complex nature. However, comparative studies have shown that LDI is a far more accurate assessment tool [15]. Yet, due to significantly lower costs, thermal imaging is used more than LDI, both clinically and in research [13]. Thermal imaging techniques are also preferred due to their ease-of-use, instantaneous image production, and device portability [16]. The primary limitation arises from the fact that the image produced by thermal imaging devices is greatly influenced by environmental factors like wound evaporation and humidity [17]. In addition, new improvements in LDI have made devices easier to move, images more accurate, and faster scanning times [18]. LDI was developed for the objective measurement of perfusion in indeterminate-depth burns to better assess the need for surgery. Despite these clear advantages, the integration of LDI into US burn centers in not nearly as widespread as those in the UK, primarily due to cost [19]. This is further supported by our survey, which indicates that not many burn centers utilize LDI nor do they plan to. A thorough financial assessment of LDI devices was performed in the Netherlands and it showed that with an initial device investment of $€ 57,590$ $(\$ 63,570)$, an average of $€ 875$ (\$1080) per patient could potentially be saved due to decreased hospital stay [20]. We contacted Moor Instruments (Axminster, UK), the manufacturer and distributor of LDI imaging systems in the UK, and an internal representative reported that a single LDI device could be purchased for as little as $\$ 60,000$, which could generate significant hospital savings. In addition to reducing hospital costs, the use of LDI in indeterminate-depth burn assessment should improve the patient experience by decreasing unnecessary surgical operations, reduced length of hospital stay, and fewer follow-up hospital visits [21,22]. At Johns Hopkins Bayview Medical Center, the estimated average direct cost of an inpatient procedure is approximately $\$ 14,000$ [23]. While we did not collect specific costs for each patient in our study, estimated expenses can help conclude that using LDI to avoid extended hospitalization and unnecessary procedures will help the hospital and the patient avoid excessive expenses.

In the United States, burn surgeons continue to use traditional approaches in managing their patients, whether it is local wound care or excision and grafting [24]. In our survey of $70 \mathrm{ABA}$-verified burns centers, only three out of 32 (9.4\%) responded confirming using LDI to evaluate burn wounds, which is slightly higher than the $6 \%$ reported in a survey from 2014 [19]. Even with this slight increase in usage, the use of LDI is significantly lower than in European burn centers (32\%) [7]. Widespread European LDI integration could be due, in part, to the official statement by the National Institute for Clinical Excellence (NICE) in the UK that LDI devices provide a clear clinical benefit in guiding clinical decisions for indeterminate-depth burns and predicting healing outcomes [25].

Medical technology is rapidly advancing in recent years with constantly emerging tools targeted for enhancing patient care. US burn centers have not been quick to adapt to new technologies even in light of their added benefits, which ultimately hinders patient care. The need for novel adjunct interventions is crucial, especially when evaluating indeterminate burns. Due to their indeterminate nature, they are significantly more complex, requiring more time for clinical assessment, which leads to a delay-to-operation, resulting in a longer hospital stay [26-28]. When preceded by LDI, surgical intervention for indeterminatedepth burn injuries allows for a more accurate course of treatment and ultimately a more positive patient experience $[29,30]$. From our experiences using LDI in the clinical setting, we can confidently confirm that the increased clinical efficiency that results from adjunct LDI-use is beneficial to both the hospital and the patient.

Our study carries a few limitations including the assumption that the two senior burn surgeons made correct and accurate decisions in managing their burn patients; their professional decision is still subjective relative to their experience. While the expert opinion of the senior burn surgeons was considered standard of care, 22 non-surgeons evaluated images because it is crucial to demonstrate that LDI-use is effective even when used by non-physician medical personnel. In most burn centers, the patient is treated by a multidisciplinary team and the technology at hand should be suited for that staff. The effect of LDI utilization on costeffectiveness, patient-care, and safety has not been thoroughly examined but we hope that this study will prompt further examination. Our study is a single-center study but we plan to expand this type of research to additional burn centers across the United States. The plan for the future is a multi-institutional study and other LDI institutions have been contacted for future prospective studies. We estimate that once these outcomes are further assessed on a nation-wide approach, the positive outcomes will pave the way for advancing the field of burn surgery in the United States. However, we are aware that every medical 
institution manages its financial budget independently so we cannot infer what calculations are considered in evaluating large expenses. Unfortunately, given the complex nature of burn wounds and the patient population involved, no proper follow-up assessing healing outcomes was performed. Finally, a technical limitation exists given that when analyzing digital photographs, there is no imaging quality standard in practice. This means that some of the images analyzed were captured by different devices at different quality levels.

\section{Conclusions}

Color-coded LDI images aid in the clinical judgment of digital photographs and make the clinical examination of indeterminate-depth burn wounds more accurate. As observed, through the utilization of LDI technology as a supplemental analysis tool, the operative plan was changed from surgical to nonsurgical intervention. For every five LDI evaluations performed, one assessor changed their treatment plan as a result of the LDI. This means that approximately $80 \%$ of surgeries proceeded as originally planned. Other studies have demonstrated that LDI is more cost-effective and is more accurate than competing imaging techniques like thermal imaging. Our study showed that digital photos alone are unreliable in predicting burn severity and the need for excision. LDI, as a supplemental tool, is cost-effective because, based on estimated patient charges and hospital expenses that can be avoided, LDI devices will pay for themselves very quickly and will increase the accuracy of determining the severity of burn wounds while significantly improving patient outcomes.

\section{Additional Information \\ Disclosures}

Human subjects: Consent was obtained by all participants in this study. The Institutional Review Board at The Johns Hopkins Hospital issued approval IRB\#00188135. The study was approved by the institutional review board at The Johns Hopkins Hospital, IRB\#00188135. Animal subjects: All authors have confirmed that this study did not involve animal subjects or tissue. Conflicts of interest: In compliance with the ICMJE uniform disclosure form, all authors declare the following: Payment/services info: All authors have declared that no financial support was received from any organization for the submitted work. Financial relationships: All authors have declared that they have no financial relationships at present or within the previous three years with any organizations that might have an interest in the submitted work. Other relationships: All authors have declared that there are no other relationships or activities that could appear to have influenced the submitted work.

\section{References}

1. Lee KC, Joory K, Moiemen NS: History of burns: the past, present and the future. Burns Trauma. 2014, 2:169-180. 10.4103/2321-3868.143620

2. Wallace DL, Jones SM, Milroy C, Pickford MA: Telemedicine for acute plastic surgical trauma and burns . J Plast Reconstr Aesthet Surg. 2008, 61:31-36. 10.1016/j.bjps.2006.03.045

3. Atiyeh B, Dibo SA, Janom HH: Telemedicine and burns: an overview. Ann Burns Fire Disasters. 2014, 27:8793.

4. Voran D: Telemedicine and beyond. Mo Med. 2015, 112:129-135.

5. Knobloch K, Rennekampff HO, Vogt PM: Cell-phone based multimedia messaging service (MMS) and burn injuries. Burns. 2009, 35:1191-1193. 10.1016/j.burns.2009.03.012

6. Sen CK, Ghatak S, Gnyawali SC, Roy S, Gordillo GM: Cutaneous imaging technologies in acute burn and chronic wound care. Plast Reconstr Surg. 2016, 138:119S-28S. 10.1097/PRS.0000000000002654

7. Rousseau AF, Massion PB, Laungani A, Nizet JL, Damas P, Ledoux D: Toward targeted early burn care: lessons from a European survey. J Burn Care Res. 2014, 35:e234-e239. 10.1097/BCR.0000000000000027

8. Jan SN, Khan FA, Bashir MM, et al.: Comparison of laser doppler imaging (LDI) and clinical assessment in differentiating between superficial and deep partial thickness burn wounds. Burns. 2018, 44:405-413. 10.1016/j.burns.2017.08.020

9. ABA Burn Center Regional Map. American Burn Association Website . http://ameriburn.org/publicresources/burn-center-regional-map/.

10. Jackson DM: The evolution of burn treatment in the last 50 years . Burns. 1991, 17:329-334. 10.1016/03054179(91)90050-Q

11. Zuo KJ, Medina A, Tredget EE: Important developments in burn care. Plast Reconstr Surg. 2017, 139:120138. 10.1097/PRS.0000000000002908

12. Monstrey SM, Hoeksema H, Baker RD, et al.: Burn wound healing time assessed by laser doppler imaging. Part 2: Validation of a dedicated colour code for image interpretation. Burns. 2011, 37:249-256. 10.1016/j.burns.2010.08.013

13. Burke-Smith A, Collier J, Jones I: A comparison of non-invasive imaging modalities: infrared thermography, spectrophotometric intracutaneous analysis and laser doppler imaging for the assessment of adult burns. Burns. 2015, 41:1695-1707. 10.1016/j.burns.2015.06.023

14. Lawson RN, Wlodek GD, Webster DR: Thermographic assessment of burns and frostbite . Can Med Assoc J. 1961, 84:1129-1131.

15. Wearn C, Lee KC, Hardwicke J, et al.: Prospective comparative evaluation study of laser doppler imaging and thermal imaging in the assessment of burn depth. Burns. 2018, 44:124-133. 10.1016/j.burns.2017.08.004

16. Medina-Preciado JD, Kolosovas-Machuca ES, Velez-Gomez E, Miranda-Altamirano A, Gonzalez FJ: Noninvasive determination of burn depth in children by digital infrared thermal imaging . J Biomed Opt. 2013, 18:061204. 10.1117/1.JBO.18.6.061204

17. Anselmo VI, Zawacki BE: Effect of evaporative surface cooling on thermographic assessment of burn depth . Radiology. 1977, 123:331-332. 10.1148/123.2.331 
18. Opazo Saez AM, Mosel F, Nurnberger J, et al.: Laser doppler imager (LDI) scanner and intradermal injection for in vivo pharmacology in human skin microcirculation: responses to acetylcholine, endothelin-1 and their repeatability. Br J Clin Pharmacol. 2005, 59:511-519. 10.1111/j.1365-2125.2004.02344.x

19. Resch TR, Drake RM, Helmer SD, Jost GD, Osland JS: Estimation of burn depth at burn centers in the united states: a survey. J Burn Care Res. 2014, 35:491-497. 10.1097/BCR.0000000000000031

20. Hop MJ, Stekelenburg CM, Hiddingh J, et al.: Cost-effectiveness of laser doppler imaging in burn care in the netherlands: a randomized controlled trial. Plast Reconstr Surg. 2016, 137:166-176. 10.1097/PRS.0000000000001900

21. Kim LH, Ward D, Lam L, Holland AJ: The impact of laser doppler imaging on time to grafting decisions in pediatric burns. J Burn Care Res. 2010, 31:328-332. 10.1097/BCR.0b013e3181d0f572

22. Sainsbury DC: Critical evaluation of the clinimetrics of laser doppler imaging in burn assessment . J Wound Care. 2008, 17:193-198. 10.12968/jowc.2008.17.5.29155

23. Johns Hopkins Bayview Medical Center. Estimated charges for inpatient procedures . (2017). Accessed: May 2020:

https://www.hopkinsmedicine.org/johns_hopkins_bayview/_docs/planning_your_visit/JHBMC_EstimatedCharges_Inpatient.F

24. Rowan MP, Cancio LC, Elster EA, et al.: Burn wound healing and treatment: review and advancements. Crit Care. 2015, 19:243. 10.1186/s13054-015-0961-2

25. moorLDI2-BI: a laser doppler blood flow imager for burn wound assessment . (2017). Accessed: May 2020: https://www.nice.org.uk/Guidance/MTG2.

26. Singer AJ, Boyce ST: Burn wound healing and tissue engineering. J Burn Care Res. 2017, 38:e605-e613. 10.1097/BCR.0000000000000538

27. Park YS, Choi YH, Lee HS, et al.: The impact of laser doppler imaging on the early decision-making process for surgical intervention in adults with indeterminate burns. Burns. 2013, 39:655-661. 10.1016/j.burns.2012.09.004

28. Ida T, Iwazaki H, Kawaguchi Y, et al.: Burn depth assessments by photoacoustic imaging and laser doppler imaging. Wound Repair Regen. 2016, 24:349-355. 10.1111/wrr.12374

29. Hoeksema H, Van de Sijpe K, Tondu T, et al.: Accuracy of early burn depth assessment by laser doppler imaging on different days post burn. Burns. 2009, 35:36-45. 10.1016/j.burns.2008.08.011

30. Khatib M, Jabir S, Fitzgerald O'Connor E, Philp B: A systematic review of the evolution of laser doppler techniques in burn depth assessment. Plast Surg Int. 2014, 2014:621792. 10.1155/2014/621792 